OPEN

SUBJECT AREAS:

ANIMAL BEHAVIOUR

BEHAVIOURAL ECOLOGY

Received

2 August 2013

Accepted

6 February 2014

Published

26 February 2014

Correspondence and requests for materials should be addressed to R.O. lokd@kph.bunriu.ac.jp)

\section{Error in the Honeybee Waggle Dance Improves Foraging Flexibility}

\author{
Ryuichi Okada', Hidetoshi lkeno², Toshifumi Kimura ${ }^{2}$, Mizue Ohashi $^{2}$, Hitoshi Aonuma ${ }^{3}$ \& Etsuro lto'
}

'Kagawa School of Pharmaceutical Sciences, Tokushima Bunri University, 1314-1 Shido, Sanuki, Kagawa 769-2193, Japan,

${ }^{2}$ School of Human Science and Environment, University of Hyogo, 1-1-12 Shinzaike-Honcho, Himeji, Hyogo 670-0092, Japan,

${ }^{3}$ Research Institute for Electronic Science, Hokkaido University, Kita 12, Nishi 7, Sapporo, Hokkaido 060-0812, Japan.

The honeybee waggle dance communicates the location of profitable food sources, usually with a certain degree of error in the directional information ranging from $10-15^{\circ}$ at the lower margin. We simulated one-day colonial foraging to address the biological significance of information error in the waggle dance. When the error was $30^{\circ}$ or larger, the waggle dance was not beneficial. If the error was $15^{\circ}$, the waggle dance was beneficial when the food sources were scarce. When the error was $10^{\circ}$ or smaller, the waggle dance was beneficial under all the conditions tested. Our simulation also showed that precise information $\left(0-5^{\circ}\right.$ error $)$ yielded great success in finding feeders, but also caused failures at finding new feeders, i.e., a high-risk high-return strategy. The observation that actual bees perform the waggle dance with an error of $10-15^{\circ}$ might reflect, at least in part, the maintenance of a successful yet risky foraging trade-off.

$\mathrm{N}$ oise and error often decrease the accuracy of biological systems such as communication, signal-detection, or system-regulation and disrupt their potential performance. The honeybee (Apis mellifera) communicates with its nestmates via the so-called "waggle dance," by which it transfers locational information about profitable food sources already visited ${ }^{1}$. Bees in close proximity to the dancing bee (followers) receive this information, and some of them may be recruited to the same food source. On the vertical comb, the direction to a food source from the hive relative to the sun's azimuth is encoded according to the angle between an upward direction and the direction in which the dancing bee walks with her body waggling (waggle run) ${ }^{1-3}$. The distance from hive to food source is related to the duration of the waggle $\operatorname{run}^{1-3}$. However, a number of studies have demonstrated some degree of imprecision in the directional information included in the dances ${ }^{4-8}$. Errors (variation) from the mean of the waggle angles have been observed in a series of waggle runs by a single dancing bee, as well as between individuals (Fig. 1a,b). Most of these errors ranged between $10-15^{\circ 9-11}$ at the lower margin, and this range is non-negligible based on the bee's broad foraging area, which can exceed $6 \mathrm{~km}^{12}$. Furthermore, the natural foraging environment changes dynamically within a single day; for example, the distribution of nectar-flowing plants and the volume of nectar differ between morning and afternoon ${ }^{13-15}$. In spite of these non-negligible errors, however, a bee colony can effectively collect food ${ }^{16-19}$ and respond quickly to changes in the profitability of food sources ${ }^{20}$ which may be defined by their distance from the hive and their concentration of food. In addition, the degree of imprecision has been shown to decrease with increasing distance to a target ${ }^{4-6}$ and also to be smaller when advertising a new nest site than when advertising a food source even if the distance is the $s^{2} e^{5}$, implying that bees may selectively benefit from responding to noisy information. Therefore, it has remained controversial whether the imprecision of the waggle dances represents an evolutional adaptation, or a full or partial physical constraint ${ }^{2,4,21-24}$.

If the range of error in the actual bee dances (most often measured by $10-15^{\circ}$ ) is of evolutionary biological significance, then the waggle dance having this degree of error must have at least two functions: first, the waggle dance must confer an advantage for food collection in a dynamically changing environment, and second, the waggle dance must permit bees to reliably respond to changes in the foraging environment. Here, we created an individual-based Markov model of Apis dance-guided foraging (iMoAD-f) and used it to perform simulations of the dance-guided foraging activity of a honeybee colony. We will propose that this degree of the error should be of the evolutionarily significant in terms of those functions.

\title{
Results
}

Measurement of parameters and modeling. We categorized the foraging-related behavior of an individual bee into eight behavioral states to create the iMoAD-f (Fig. 1c). Transitions between the behavioral states were 

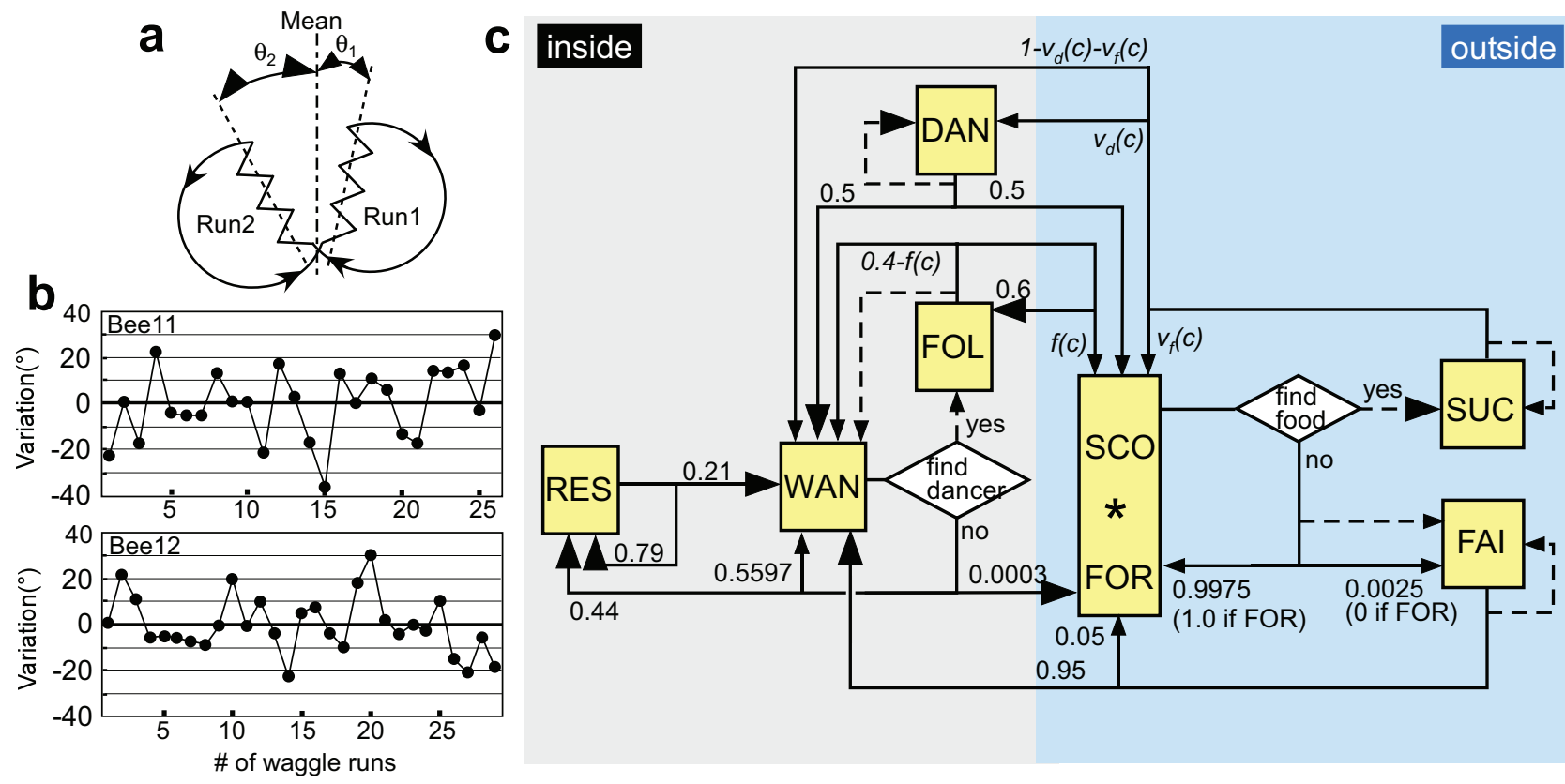

Figure 1 The waggle dance and our model. (a), Variation of directional information encoded by waggle runs. Each run had a certain degree of variation from the mean waggle-run direction. (b), Sequences of information variation in consecutive waggle runs of two dancers (Bee 11 and Bee 12). The mean direction is represented as $0^{\circ}$. (c), iMoAD-f. Eight behavioral states are indicated and transitioned by a specific probability and state-specific rules. For visualization, scouting (SCO) and foraging (FOR) are represented in the same box, because these two states were essentially the same, except that a bee could be flying in search of a feeder (i.e., lacking directional information about a feeder), or it could be flying to a known feeder (i.e., with directional information about the feeder). The numbers beside the solid lines indicate the transition probabilities (see Tables 1 and 2). Dotted lines indicate that the states automatically transitioned, i.e., the transition probability was 1, under state-specific rules (see Supplementary Methods). The probability of scouting from success $\left(\mathrm{V}_{\mathrm{f}}(\mathrm{c})\right)$, of dancing from success $\left(\mathrm{V}_{\mathrm{d}}(\mathrm{c})\right)$, and of foraging from following $(\mathrm{f}(\mathrm{c}))$ depended on the concentration of feeders (see Table 2). DAN, dancing; FAI, failure; FOL, following; FOR, foraging; RES, resting; SCO, scouting; SUC, success; WAN, wandering.

determined by the probabilities discussed in the literature ${ }^{25}$ (Table 1 ) and by state-specific rules (see Supplementary Methods). Importantly, probabilities related to dancing and following depended on the concentration of sugar solution in the feeder (Table 2), based on previous observations $\mathrm{s}^{20,26,27}$.

For parameterization of the simulations, we first videotaped the bee behavior, and then extracted and measured the parameters (Supplementary Fig. S1 and Supplementary Table S1). We found that dancing was performed in a restricted area, the so-called dance floor (Supplementary Fig. S1a). About $85 \%$ of followers (476 follows from 414 followers) turned away from their dancer after one or two waggle runs (Supplementary Fig. S1b). Walking bees also tended to be found on the dance floor (Supplementary Fig. S1c). Walkers moved forward but not far, i.e., at most $2.0 \mathrm{~cm} / \mathrm{s}$ (Supplementary Fig. S1d,e,f), and changed their body axis between $-40^{\circ}$ and $40^{\circ}$ (about $80 \%$ of 19331 -sec walks) (Supplementary Fig. S1g). The body weights of bees with a crop full of nectar and bees with an empty crop $($ mean \pm SE) were $119 \pm 2.75 \mathrm{mg}(\mathrm{n}=10)$ and $78.5 \pm 1.62 \mathrm{mg}(\mathrm{n}=$ $14)$, respectively. The bees with full crops were likely to be successful foragers, and those with empty crops to be failed foragers. About $85 \%$ (304 out of 358 runs) of directional information variation ranged within $15^{\circ}$, as we previously observed ${ }^{9,10}$. In addition to our own present and previous results, results from the literature were used to run the simulation (Supplementary Table S1). Most importantly, only measurable parameters were used, and thus our simulation results might be evaluated in future biological experiments.

Evaluation of the model. We evaluated iMoAD-f by comparison with a well-known experiment by Seeley et al. $^{20}$, which is often used as a framework for modeling honeybee foraging ${ }^{28-32}$. In

Table 1 | Transition probabilities between two behavioral states

next behavioral state

\begin{tabular}{|c|c|c|c|c|c|c|c|c|}
\hline & Res & Wan & Sco & For & Suc & Fai & Dan & Fol \\
\hline Wan & 0.44 & 0.5597 & $0.0003^{a}$ & $0.0003^{a}$ & - & - & - & $1.0^{\mathrm{b}}$ \\
\hline For & - & - & - & $1.0^{\mathrm{b}}$ & $1.0^{\mathrm{b}}$ & $1.0^{\mathrm{b}}$ & - & - \\
\hline Suc & - & variable & - & variable & $1.0^{\mathrm{b}}$ & - & variable & - \\
\hline Fai & - & 0.95 & $0.05^{a}$ & $0.05^{a}$ & - & $1.0^{\mathrm{b}}$ & - & - \\
\hline Dan & - & 0.5 & - & 0.5 & - & - & $1.0^{\mathrm{b}}$ & - \\
\hline
\end{tabular}


Table 2 | Concentration-dependent transition probabilities of dancing and following

\begin{tabular}{|c|c|c|c|c|c|c|}
\hline & \multicolumn{3}{|c|}{$\begin{array}{l}\text { Successful flight } \\
\text { Concentration (M) }\end{array}$} & \multicolumn{3}{|c|}{$\begin{array}{c}\text { Following } \\
\text { Concentration (M) }\end{array}$} \\
\hline & 0.75 & 1.0 & 2.5 & 0.75 & 1.0 & 2.5 \\
\hline $\begin{array}{l}\text { Dancing } \\
\text { Foraging } \\
\text { Wandering } \\
\text { Following }\end{array}$ & $\begin{array}{c}0.15^{a} \\
0.13^{c} \\
0.72^{c} \\
-\end{array}$ & $\begin{array}{l}0.26^{a} \\
0.14^{c} \\
0.6^{c} \\
-\end{array}$ & $\begin{array}{l}1.0^{a} \\
0^{a} \\
0^{a} \\
-\end{array}$ & $\begin{array}{l}0 . \\
0.05^{c} \\
0.35^{c} \\
0.6^{b}\end{array}$ & $\begin{array}{l}0 . \\
0.05^{c} \\
0.35^{c} \\
0.6^{b}\end{array}$ & $\begin{array}{l}{ }^{-} \\
0.1^{c} \\
0.3^{c} \\
0.6^{b}\end{array}$ \\
\hline
\end{tabular}

Seeley's experiment, two feeders were placed $400 \mathrm{~m}$ away from the hive to the south and the north, respectively. The concentrations of sugar water in the south and north feeders were $2.5 \mathrm{M}$ and $1.0 \mathrm{M}$, respectively. After 4 hours, the concentrations were changed to $0.75 \mathrm{M}$ and $2.5 \mathrm{M}$, respectively. They found that the colony altered its feeder visitation habits in response to the change in the concentration of sugar water. We reproduced Seeley's experiment, and 20 simulations yielded similar results (Supplementary Fig. S2). Thus, we concluded that iMoAD-f reliably expressed the behavior of an actual bee colony.

Availability of the waggle dance. Do waggle dances really confer an advantage for food collection? To examine the first function, we compared the foraging results among four virtual colonies characterized by their foraging strategies: a random-search colony, a nocommunication colony, a random-information dance colony, and a normal waggle dance colony (see Methods). Twenty simulations of one-day foraging by a 1000 -bee colony under experimental conditions mimicking those of Seeley et al. revealed a large difference in the total number of visits to all feeders for the entire foraging time among colonies (Fig. 2a). For the random-search colony, no-communication, random-information dance and waggle dance colonies, the mean total numbers of visits, with standard deviations, were $409.5 \pm 14.1,1348.1 \pm 75.5,1978.0 \pm 292.3$, and $2756.4 \pm 237.0$, respectively. The analysis of variance (ANOVA) showed a significant effect of colony type $(\mathrm{P}<0.001)$. Among the colonies tested, the waggle dance colony made a significantly greater number of successful visits to food sources than the other three colonies (Fig. 2a, $\mathrm{P}<0.001$ for all combinations, Tukey-Kramer test).

For evaluating foraging success, the energy-balance results are more general and useful than the number of visits, because a gain in energy will enhance the survivability of the colony. We calculated the energy balance by subtracting the energy loss from the energy gain for one-day foraging using the values in Figure 2a and found that the waggle dance colony gave the best foraging performance, as expected (Fig. 2b). Interestingly, there were no longer significant differences in energy balance between the no-communication and random-information dance colonies under the present conditions (Fig. 2b). Another 100 simulations under the same conditions as used in Figure 2a, i.e., 2 feeders at $400 \mathrm{~m}$, demonstrated that the rates of success expressed as a percentage of total visits of the two colonies were almost identical (38.6\% for the no-communication colony and $38.5 \%$ for the random-information dance colony), i.e., the efficiency was the same (Supplementary Fig. S3).

When the number of feeders was increased from 2 to 10 , the waggle dance colony showed the greatest energy gain, but this result did not differ significantly from that for the random-information dance colony (Fig. 2c), indicating that both the informative and the non-informative dances performed best under this feeder condition. These findings suggest that the best foraging strategy is dependent on the number of feeders.
Optimal conditions for dance execution. We then examined the relationship between the spatial distribution of feeders and the efficiency of the waggle dance as assessed by energy balance, taken together with variations in directional information. iMoAD-f revealed that either informative or non-informative dancing behavior was beneficial in 139 (82.7\%) of 168 tested conditions (Fig. 2d), suggesting that interactions between individuals per se are important for collective foraging, irrespective of the information exchanged. In addition, random searching may not be strategic, as no best-cases were found in this group (Fig. 2d).

As shown in Figure 2c, the informative dance (waggle dance colony) was not always beneficial (Fig. 2d). When the variation was $5^{\circ}$ or less, only the waggle dance colony was the best colony (colonies with a performance not significantly different from the best colony were also classified as best colonies; see Methods) under all conditions tested (Fig. $2 \mathrm{~d}$ ). When the variation was $10^{\circ}$, the waggle dance colony remained the best performer, and there were cases in which the random-information dance colony and/or the no-communication colony performed equally well (Fig. $2 \mathrm{~d}$ ). Under this variation $\left(10^{\circ}\right)$, only the waggle dance colony was the best colony if there were a small number of feeders, which suggested that the feeders were hard for the bees to locate. In contrast, when the variation was large $\left(30^{\circ}\right.$ or $\left.60^{\circ}\right)$, the waggle dance colony did not perform the best. Interestingly, when the variation was $15^{\circ}$, the results were highly mosaic (Fig. 2d). Basically, when the feeders were not easy to find due to the small number of feeders, the waggle dance colony performed the best, and the random-information dance colony performed the best when the feeders were easy to find. The no-communication colony performed the best when the feeders were far away $(2000 \mathrm{~m})$. If the bees persisted with informative dancing when the feeders were at this latter distance, the variation had to be $10^{\circ}$ or less (Fig. 2d). Otherwise the colony could not expect an advantage from dancing. Consistently, our video analysis of the actual dance behavior yielded the same conclusions as the simulation analysis (Fig. 2e), i.e., the variation in directional information was reduced as the distance from the hive to the feeder increased. In summary, iMoAD-f suggested that a variation of $15^{\circ}$ is the upper limit for gaining a foraging advantage from informative dancing.

Switching feeders. Although Figure $2 \mathrm{~d}$ indicates that a colony always gained an advantage from dancing when the variation was below $5^{\circ}$, it does not explain why real bees dance with an error of $15^{\circ}$ (just below the upper limit) or larger. In other words, does a variation of $15^{\circ}$ have any biological significance? To answer this question, we next examined the other function, i.e., the adaptability of a colony to change in an environment, by focusing on the time course of the number of visits to each feeder. The time course showed a clear switch in the feeders visited by the waggle dance colony, but not by any of the other colonies (Fig. 2f). The random-search colony, nocommunication colony, and random-information dance colony never ceased visiting the low concentration feeders. 

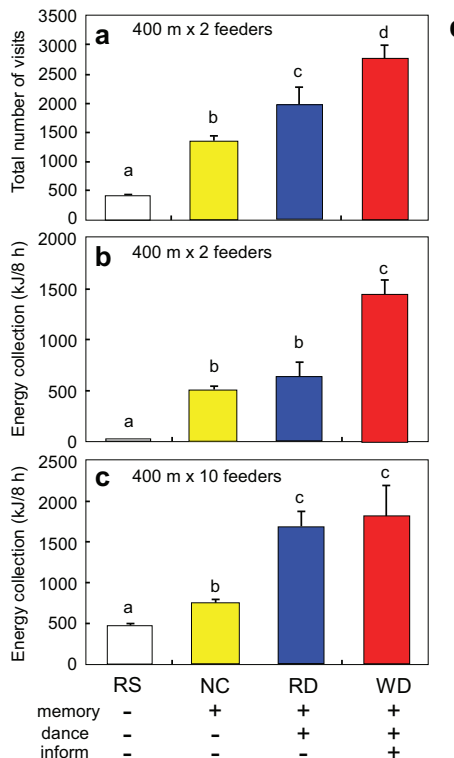

d
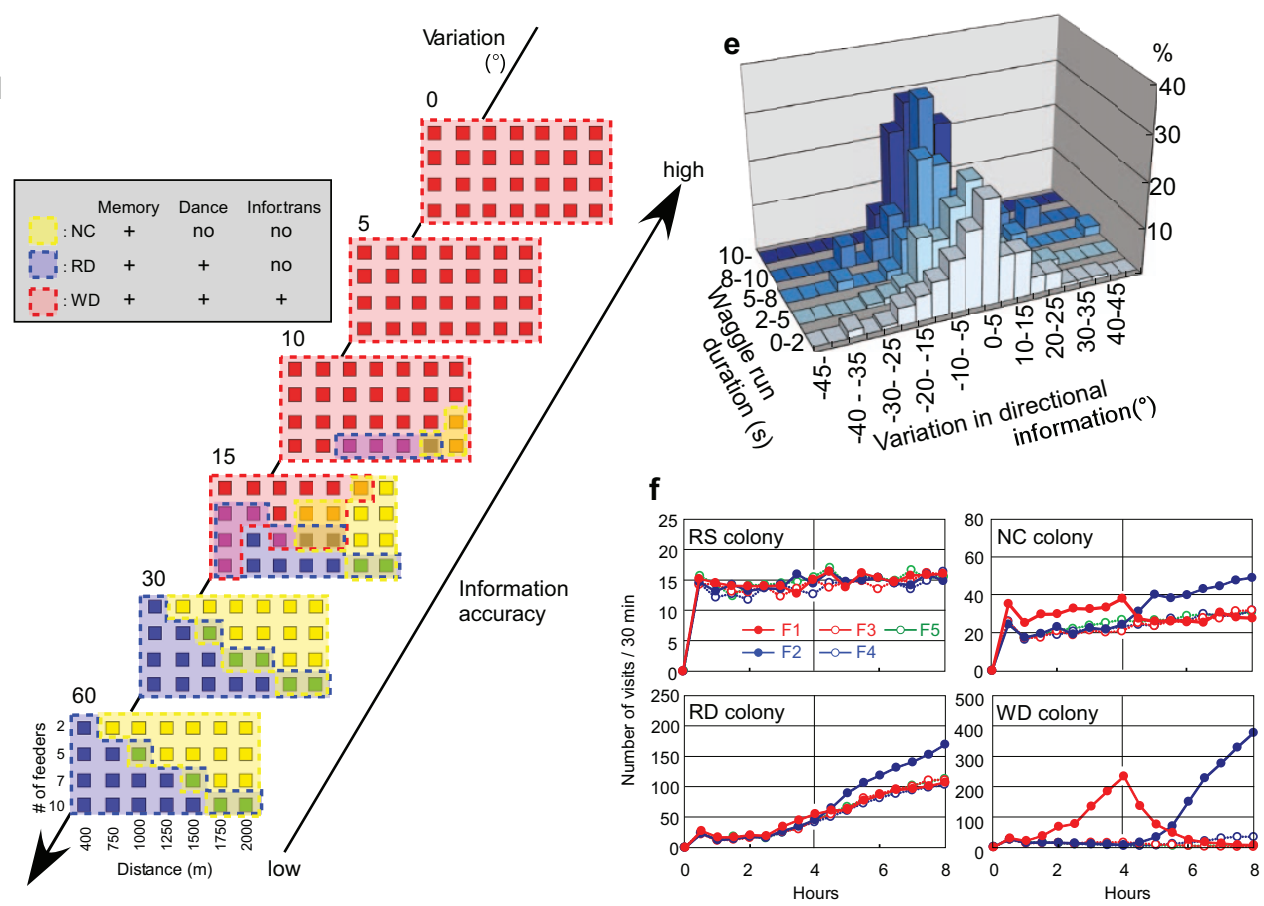

Figure $2 \mid$ Availability of the waggle dance. Comparisons of four virtual colonies in terms of visits to feeders (a) and colony economics (b and c). The letters above the boxes represent groups showing statistically significant differences (ANOVA and post-hoc Tukey-Kramer test). Interestingly, there was no significant difference in colony economics between the no-communication colony and random-information dance colony (b), even though these two colonies exhibited a significant difference in the total number of feeder visits (a), even under the same conditions. The waggle dance colony did not always outperform the other groups (c), depending on feeder distribution. (d), Summary of an energetically advantageous colony (indicated by color codes) with the tested feeder distribution and the degree of variation. Under some conditions tested, there were cases in which more than one colony was not significantly different from the best colony. In such cases, the indicated colors are mixed with the corresponding colony's colors. Outlines for each colony are also shown. In each variation panel, the upper-right corner represents the most difficult feeder location distribution (the smallest number of feeders and also the longest distance from the hive); the bottom-left corner represents the easiest feeders to locate. (e), Variation distribution dependent on the waggle run duration (corresponding to the distance from the hive) during the performance of actual bees. The variation became smaller with an increase in the waggle run duration. (f), Examples of the time course for the number of visits for the four colony types. The five feeders were distributed $400 \mathrm{~m}$ away from the hive, with $15^{\circ}$ variation. Note that the $y$-axis differed among the four colonies. Only the waggle dance colony exhibited clear switching of feeders, i.e., from feeder 1 to feeder 2, after changes were made to the sucrose concentration in each feeder. NC, no-communication colony; RD, randominformation dance colony; RS, random-search colony; WD, waggle dance colony. F1-5, feeders 1-5.

We calculated the rate of visits (see Methods), and found that in phase 1 (at 4 hours), the waggle dance colony had clearly visited the currently more profitable feeder (Fig. 3a, b), when the two feeders were available. Other colonies did not show such distinct behavior, although there were slightly higher visit rates for the more profitable feeder. In the case of the larger variations $\left(30^{\circ}\right.$ and $\left.60^{\circ}\right)$, the visit rate for the more profitable feeder by the waggle dance colony was lower than that of the other variations at each of the distances (Fig. 3a, b). When five or more feeders were made available, the visit rates for the most profitable feeder in phase 1 were more intensive than those for any other feeders (Fig. 3b). In phase 2 (at 8 hours), clearly higher visit rates for the most profitable feeder (feeder 2 ) by the waggle dance colony were seen, but no distinct differences were noted with the other colonies. When the number of feeders increased, the correct responses to the change in feeder concentration were observed only in the waggle dance colony (Figs. $2 \mathrm{f}$ and $3 \mathrm{~b}$ ). Detailed observations, however, revealed cases in which the waggle dance colony failed to switch (Fig. 3c and d). Specifically, increases in both the distance to the feeder and variation were associated with a higher number of failures (Fig. 3c).

Quantitative analysis of switching. To quantify the rate of success in switching the visited feeder, further analysis was carried out using only the waggle dance colony with a variation of $0-15^{\circ}$, because iMoAD-f suggested that only the waggle dance colony could perform the switch and because iMoAD-f revealed that the waggle dances were not advantageous when the variation was $30^{\circ}$ or $60^{\circ}$. We calculated the switching index (see Methods) for all runs tested. The bees exhibited good switching, i.e., a high switching index, when the feeders were located $400 \mathrm{~m}$ or $750 \mathrm{~m}$ (Fig. 4) away from the hive, regardless of the number of feeders. When the feeders were 1000$1250 \mathrm{~m}$ away from the hive, negative switching indices were found in more cases than under the shorter-distance conditions (Fig. 4). Surprisingly, negative switching indices were seen even when the variation was $0^{\circ}$, although positive switching indices were also observed under these conditions. When the feeders were $1500 \mathrm{~m}$ or farther away from the hive, the bees failed to switch more often than when the feeders were closer. Strikingly, the negative switching indices were always near -100 , while the range of positive switching indices was broader. Thus, the occurrence histogram of the switching indices showed two peaks-namely, one around 80 and the other at -100 for the variation of $0^{\circ}$ or $5^{\circ}$. In contrast, when the variation was $15^{\circ}$, equal distribution (no prominent peak) was found in the histogram (Fig. 4).

For further analysis of the switching manner, we calculated the degree of advantage by waggle dances (see Methods) for each combination of variation and distances. When the variation was $0^{\circ}, 5^{\circ}$, or $10^{\circ}$, a degree of advantage of 4 was found for all combinations. When the variation was $15^{\circ}$ (Fig. 5a), a degree of advantage of 4 was found only for the $400-\mathrm{m}$ feeder condition and a degree of 0 was found only for the $2000-\mathrm{m}$ feeder condition. In the other five combinations, the degree of advantage ranged between 1 and 3 . 

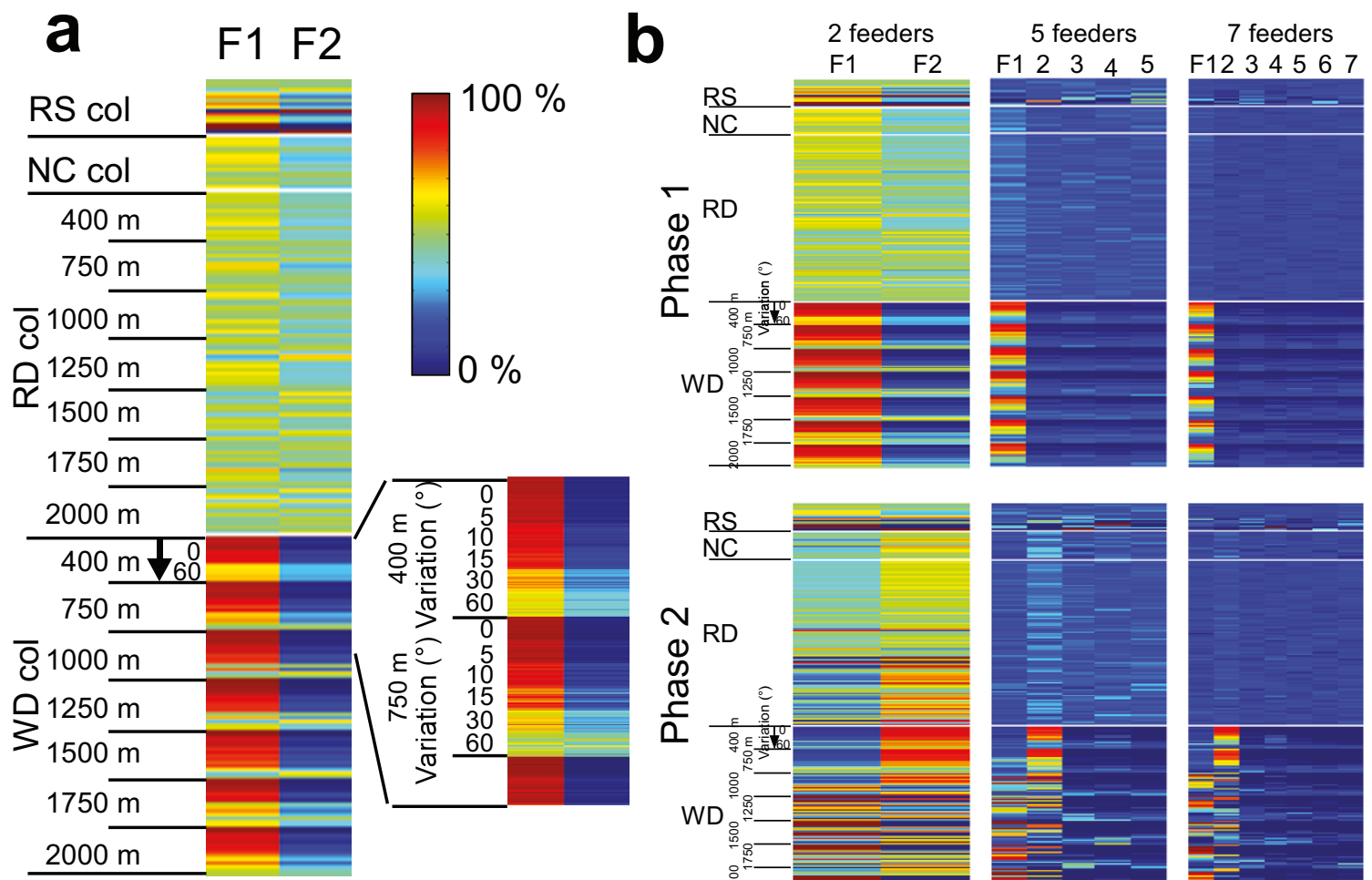

10 feeders
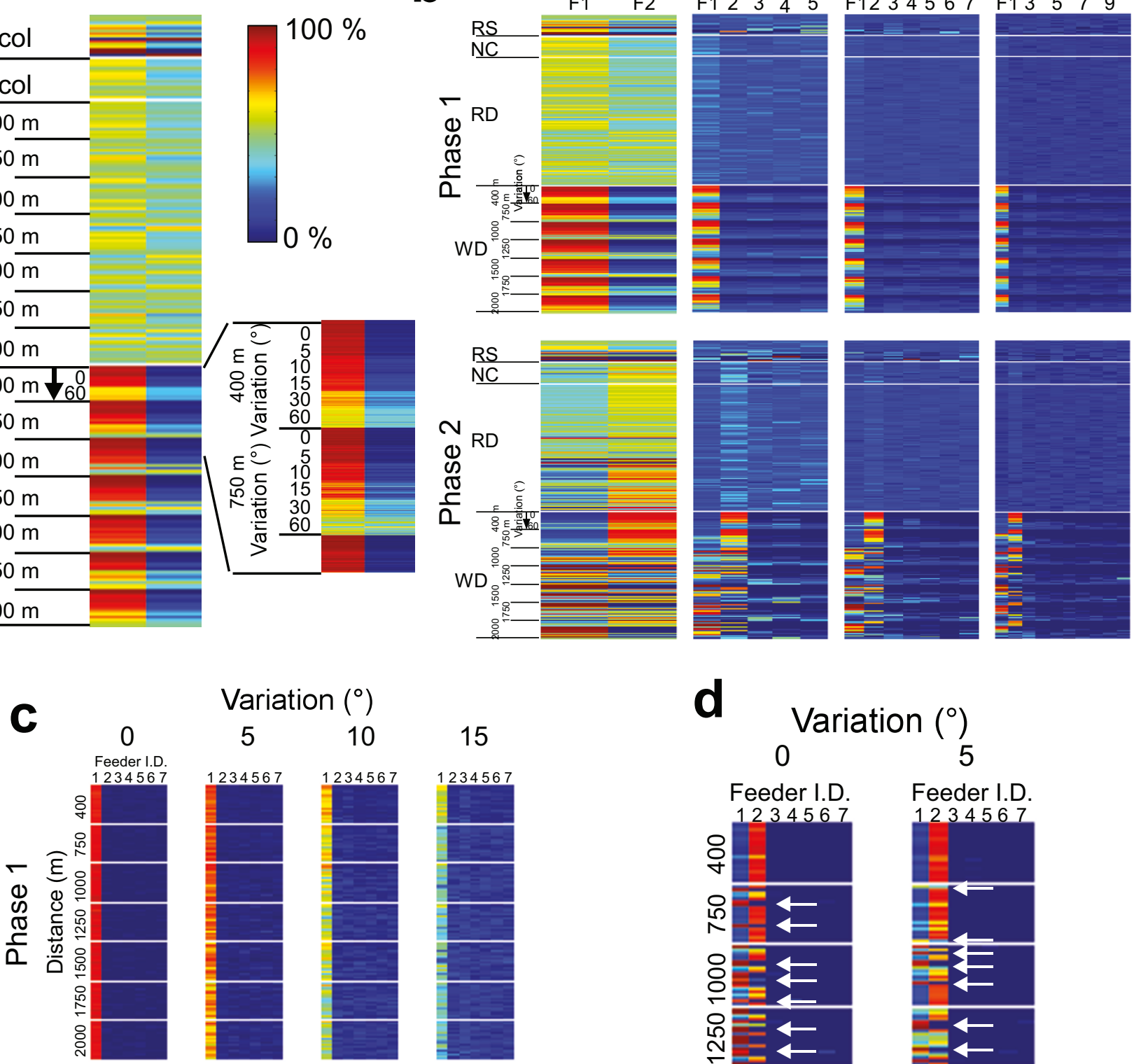

\section{5}
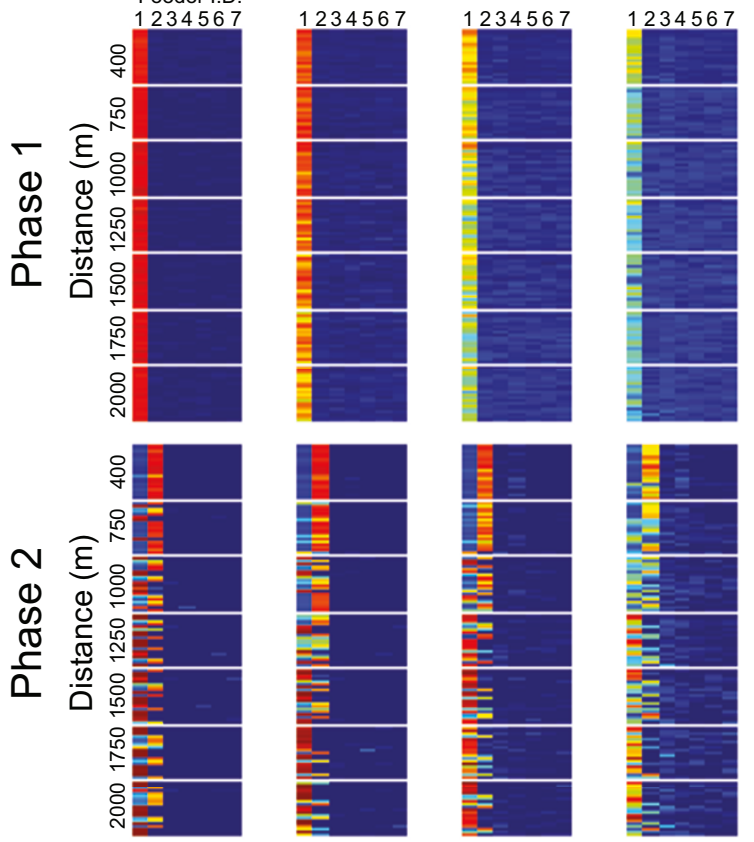
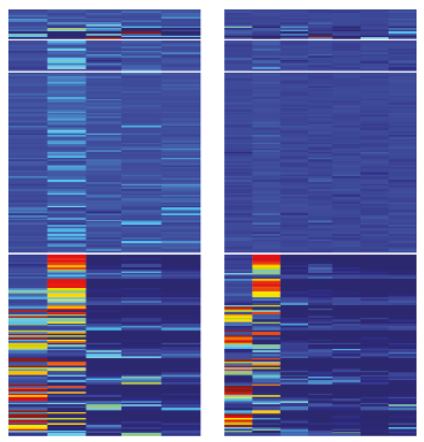

d

\section{Variation $\left({ }^{\circ}\right)$ \\ 0}

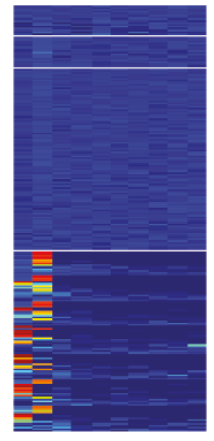

Figure 3 Visit rates coded by color. (a) and (b), visit rates of all simulation results (20 runs for each simulation condition) for the two-feeder condition in phase 1 (a) and all conditions in phase 1 and 2 (b). Only the waggle dance colony clearly showed high visit rates for feeder 1 in phase 1 , meaning that a greater number of bees in the waggle dance colony visited the most profitable feeder and that bees in other colonies visited each of the feeders with almost equal frequency. In phase 2, the random-search colony, no-communication, and random-information dance colonies showed almost no switching, although the random-information dance colony exhibited some switching under the two-feeder conditions. Thus, only the waggle dance colony succeeded at switching visited feeders in response to environmental changes. (c) and (d), visit rates by the waggle dance colony under 7 -feeder conditions (c) and under the variations of $0^{\circ}$ and $5^{\circ}$ with high magnification (d). In phase 1, feeder 1 attracted the largest number of visits (c). In phase 2 , although feeder 2 was expected to attract the largest number of visits, in some cases feeder 1 remained the most frequently visited feeder, indicating that switching was not successfully carried out in all cases (the white arrows in (d) indicate the representative cases). F1-10, feeder 1-10; NC, no-communication colony; $\mathrm{RD}$, random-information dance colony; RS, random-search colony; WD, waggle dance colony. 


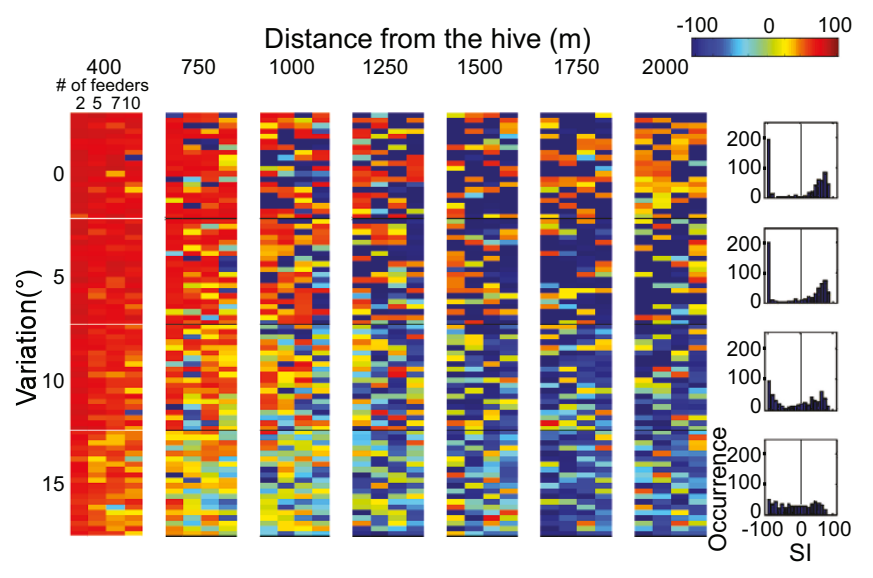

Figure $4 \mid$ Switching indices by the waggle dance colony for all simulation runs. Switching indices of all single simulation runs were color-coded. The column at the far right shows the occurrence histograms of switching indices of the corresponding row. When the error was $0^{\circ}$ or $5^{\circ}$, high-risk, high-return foraging occurred. SI: switching index.

We found that the number of negative switching indices increased as the distance to the feeders increased (Fig. 5b). The prominent failures in switching, i.e., the cases in which the switching index was nearly -100 , were pronounced when the feeders were located $1500 \mathrm{~m}$ or longer away from the hive for a variation of $10^{\circ}$ or less. When the variation was $0^{\circ}$ or $5^{\circ}$, such prominent failures in switching began to be found at a shorter distance $(1000 \mathrm{~m})$. However, when the feeders were located $400 \mathrm{~m}$ away, the switching indices usually took large positive values. Because all the combinations described above were assigned a degree of advantage of 4 , there might be no or little relationship between the degree of advantage and the switching manner. Interestingly, for a distance of $1750 \mathrm{~m}$ or longer and a variation of $5^{\circ}$ or $10^{\circ}$, the histograms showed only one peak at -100 . This means that bees very often failed to switch the visited feeder.

\section{Discussion}

We created an individual-based Markov Model of honeybee danceguided foraging, iMoAD-f. iMoAD-f was validated by several lines of results that were consistent with previous findings from biological and theoretical experiments. Most importantly, iMoAD-f expressed bee-foraging behavior similar to that in Seeley's experiment $^{20}$. It is known that information error (variation) decreases with increases in feeder distance $e^{4,5,33}$, and that colonies respond to changes in feeder conditions $s^{20}$. The waggle dance was found to be advantageous when fewer feeders were available, i.e., when the feeders were difficult to find, both in biological experiments ${ }^{17-19}$ and modeling studies ${ }^{30-32,34,35}$.

Although numerous modeling and simulation studies of honeybee foraging have been reported to date $\mathrm{e}^{28-30,34-40}$, many, if not all, have used quantitatively and physically unmeasurable parameters, e.g., homing motivation and foraging motivation ${ }^{30}$. This kind of partial lack of representation of real-world conditions has prevented a truly comprehensive understanding of the functions and mechanisms of the waggle dance. Here, we used only physically measurable parameters, which enabled us to evaluate iMoAD-f directly by using experiments with real bees. Furthermore, the iMoAD-f could be improved quickly by incorporating any additional features that we found necessary-for example, the diurnal change in variance of dance direction change in the source of the day ${ }^{41}$, characteristics of the outbound flight of the foragers with the dance information (social information) or individual memory (private memory) ${ }^{42}$, and the visiting pattern of the foragers, such as the flower constancy ${ }^{43}$ and the cross visit ${ }^{42}$. Further experiments on the followers would facilitate a
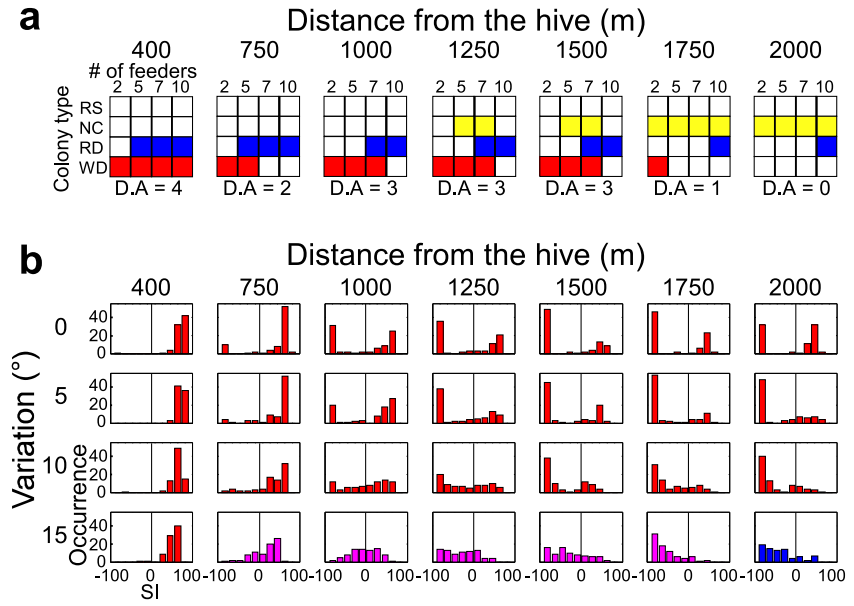

Figure $5 \mid$ Details of switching. (a), The best colonies when the error was $15^{\circ}$. (b), Occurrence histograms of switching indices for each variationdistance combination shown in Figure 4. The position of each histogram corresponds to the position in the color-coded panels shown in Figure 4. The color of each histogram indicates the degree of advantage; red, magenta, and blue histograms indicate degrees of advantage of 4, 1-3, and 0 , respectively. D.A., degree of advantage; NC, no-communication colony; $\mathrm{RD}$, random-information dance colony; RS, random-search colony; SI, switching index; WD, waggle dance colony.

improvement of iMoAD-f. It is possible that the probability of following a dancer depends on the characteristics of the external environment, such as the distance and scarcity of food sources, and the internal environment of the hive, such as the amount of stored nectar. The current version of iMoAD-f does not account for these features, mainly because a trade-off was necessary between simplicity and real-world simulation (Supplementary Discussion). However, the future incorporation of such parameters will render the iMoAD-f more realistic.

Unlike the previous models $\mathrm{s}^{2028-32,34-37}$, iMoAD-f deals with variations in the information transferred by waggle dances. This new model should thus provide new insights into the efficiency of these dances. The iMoAD-f suggests that waggle dance-mediated information transfer with the smallest variation potentially carries the risk of a complete failure to find the most profitable feeder. In other words, a particular range of error in directional information enables a colony to achieve a means of flexible foraging in a dynamically changing environment. When the same analysis was applied to energy balance, identical results were obtained (Supplementary Fig. S4). To our knowledge, this is the first report on the relationship between the manner of foraging and the accuracy of information. The iMoAD-f also suggests that if the variation was $10^{\circ}$ or less, the waggle dance colony was always at an advantage relative to the colonies using other foraging strategies regardless of the scarcity of the feeders, although the previous experiments suggested that the waggle dance is efficient when the food sources are scarce (clustered) $)^{18,19}$. In contrast, when the variation was $30^{\circ}$ or $60^{\circ}$, the waggle dance colony was no longer the most efficient. Interestingly, when the variation was under $30^{\circ}$ or $60^{\circ}$, the random-information dance colony was the most efficient when the food source was easy to find (i.e., when there were many food sources and/or they were close to the hive). This may explain, from the viewpoint of the energy balance, the fact that real bees exhibit a round dance-i.e., a dance considered not to transfer directional information-when the food is near the hive. Therefore, in future biological experiments on dance effects, the variation in information should be measured simultaneously; otherwise, the real effects of the dance might be missed. 
Noise and error are a major problem in biological systems, and often reduce the accuracy of these systems and degrade performance. Nevertheless, in the case of bee colonies, providing information with no noise/error was also not always suitable. iMoAD-f, in which the dance probability was defined only by a successful forager that evaluated the profitability of the food source, showed that dances with the smallest variation yielded the greatest number of visits to a feeder with the highest sucrose concentration (Supplementary Fig. S5). However, many foraging bees completely failed to switch feeders (Fig. 4). The finding, under the conditions we simulated, that extremely accurate directional information (overly narrow tuning) in waggle dances recruited the most bees to the advertised feeder suggested that the bees had a much lower chance of finding another feeder. Consequently, the colony had to abandon its flexible response to abrupt changes in the foraging environment.

We have shown the possible role of information error in waggle dances, but further questions must be answered for a full understanding of the function and mechanism of these dances. Does a follower bee use information from all waggle runs it attends or only from selected waggle runs? Is the dance efficiency a result of the error in dance information or simply a by-product of inaccurate dances? We believe that the improvement of a colony's flexibility by dance inaccuracy is not simply a fortunate by-product because the degree of imprecision of the directional information depends on the target ${ }^{5}$, i.e., we speculate that bees might actively exploit error for foraging. To examine this hypothesis, the variation in dance information must be controlled in a real-world setting. One possibility is to perform biological experiments using a robot bee that could recruit nestmates to a food source by the waggle dance rules ${ }^{44}$. Another possibility is to use a computer simulation. The combination of biological experiments and theoretical research will provide new insights and a fuller understanding of the waggle dance.

Noise sometimes contributes to an improvement in system performance. For example, mechanosensory neurons can detect small signals using noise $\mathrm{s}^{45-49}$. In ants, noise can improve collective decision-making ${ }^{50}$. Hence, a specific range of error in the waggle dance can be expected to be of significance. Efficient food collection is likely to have selective benefits. Food collection must consist of two major activities: acquiring food (choosing the effective foraging strategy in our work) and responding quickly to changes in the environment relating to food collection (switching the visited feeder). Although we cannot exclude the possibility of physical constraints, taken together with the dependency of precision in the directional information on the target ${ }^{4,11,22}$ and the smallest variation ranges ${ }^{9-11,24}$, our present results suggest that a $10-15^{\circ}$ variation in the conveyance of food-source information might be optimal for guaranteeing foraging success under a dynamically changing environment. Therefore, this range of error might be the result of a naturally selected trade-off between risk and success.

\section{Methods}

Video analysis. To obtain parameters for modeling and simulation, behavioral analysis was performed as described in our previous work ${ }^{9,10}$. Briefly, about 1500 honeybees, Apis mellifera, were kept with a queen in an observation box under fully natural conditions at the campus of Hokkaido University (Sapporo, Japan). The experimental location was a flower-rich area, but there were fewer flowers than in spring. This situation sufficiently induced waggle dances. There were 1-10 dancing bees at one time. The bee behavior on the vertical comb was videotaped at 30 frames/ sec (GR-HD1; JVC, Tokyo, Japan) from 8:30 am to 4:00 pm on several days in August and September, 2006 (temperature, $25-36^{\circ} \mathrm{C}$ on most days). All analyzed data were obtained off-line and frame-by-frame with a computer after conversion to JPEG image files. The locomotion of bees in the hive was analyzed by tracing the trajectories of locomotion (sampling rate $=1 \mathrm{~Hz}$ ). The dance orientation was calculated as a straight line by measuring the angle between the starting point and the ending point of a waggle run, and dance duration was calculated by counting the number of frames of video for each waggle run.

Modeling. We created an individual-based Markov model (iMoAD-f) by classifying the foraging behavior of honeybees into eight states: inactive (resting), wandering in the hive (wandering), flying while searching for a feeder (scouting), flying to return back to the hive with or without food (success or failure, respectively), flying to a feeder with directional information (foraging), dancing (dancing), and following a dancer (following). Each behavior state changed based on a given transition probability $^{25}$ (Fig. 1c and Table 1). Importantly, probabilities related to dancing and following depended on the sucrose concentration at the feeder (Table 2). Rewarddependency was responsible for the response to a change in circumstances (Supplementary Fig. S6). Other parameters for modeling and simulation were obtained from our own analysis of videotapes or from the published literature (Supplementary Fig. S1 and Supplementary Table S1). In addition to probability, states also changed according to state-specific rules. For example, if a wandering bee had moved closer to a dancing bee than the distance necessary to find a dancer (15 mm, Par-38 in Supplementary Table S1), the state of the wandering bee transitioned from wandering to following, irrespective of probability. On the other hand, if the following bee exceeded that distance, this bee was automatically considered to be wandering. These transitions were incorporated into our simulation based on our preliminary observation that most bees within one body length (ca $15 \mathrm{~mm}$ ) appeared to orient to a dancing bee reflexively, and followers appeared to lose their dancer when the dancer changed walking direction rapidly, when a follower was obstructed momentarily by another bee, or when the dancer was out of range (data not shown). For more details about modeling, see the Modeling and simulation section in the Supplementary Methods.

Simulations. A single-day (8-hour) of activity was simulated (time step $=1 \mathrm{sec}$, thus 28,800 time steps in total). The hive contained 1000 worker bees. The 2, 5, 7, or 10 feeders were located radially around the hive at 400, 750, 1000, 1250, 1500, 1750 , or $2000 \mathrm{~m}$ away from the hive. All feeders were set at the same distance in a single simulation. In the experiment with two feeders, the concentration of sucrose solution in feeder 1 was $2.5 \mathrm{M}$ and that in feeder 2 was $1.0 \mathrm{M}$ for the first 4 hours (phase 1). In the second 4-hour period (phase 2), the concentration of the sucrose solution in feeder 1 was $0.75 \mathrm{M}$ and that in feeder 2 was $2.5 \mathrm{M}$. This

concentration treatment was the same as in Seeley's experiment ${ }^{20}$, which was used for the validation of iMoAD-f. The feeders were always placed in opposite directions from the hive (Supplementary Fig. S7). In other scenarios, the sucrose concentrations in feeder 1 and feeder 2 were the same as those in the two-feeder simulations, and the sucrose concentrations in the remaining feeders (feeder 3-10) were kept constant at $1.0 \mathrm{M}$ throughout the experiment. The feeder closest to feeder 1 was designated feeder 3 , and that closest to feeder 2 among the remaining feeders was feeder 4 . Although we saw essentially the same results with a simple reverse of the concentrations when the 2 feeders were located $400 \mathrm{~m}$ away from the hive, we kept Seeley's concentration conditions in order to permit an easy comparison of results throughout all simulation experiments.

The directions of feeders from the hive were randomly distributed for every run, but the same set of 20 feeder-distribution conditions was used for the same number of feeder conditions (Supplementary Fig. S7). Thus, we were able to eliminate unexpected spatial effects of feeder distribution and investigate only the influence of distance. Feeders were always located more than $120 \mathrm{~m}$ (twice the radius of the search area for a flying bee) apart in order to prevent a flying bee from finding more than one feeder at the same time. We performed the simulation once for each set of 20 feeder distributions. To execute simulations under conditions similar to those of Seeley et al., each feeder was known by 10 bees as an initial condition.

Analysis. For the comparison with Seeley's experiment ${ }^{20}$, we excluded initiallyinformed bees from the analysis and counted only the number of recruited individuals, not visits, every 30 minutes. For other analyses, we used all 1000 bees to evaluate the benefits to an entire colony. We counted the total number of visits to all feeders in each trial, and then calculated the average number of visits. Using energetics information (Supplementary Table S1), we also calculated the colonial energy balance by subtracting the energy out from the energy in, and then compared the results among virtual colonies. The advantage of dancing behavior was examined by comparing the number of visits in feeders and energy balance over a single day among four virtual colonies as follows (for details see Supplementary Methods): a random-search colony in which bees did not memorize the location of the feeders or perform dances; a no-communication colony in which bees memorized the location of the visited feeder, but did not communicate with each other (no dance was performed); a random-information dance colony in which bees memorized the feeder location and danced, but transferred meaningless directional information about the feeder; and a waggle dance colony in which bees memorized feeder locations, danced, and transferred significant information with variations of $0,5,10,15,30$, or $60^{\circ}$. The colony with the highest value of energy balance was referred to as the best colony. If a colony or colonies were not significantly different from the best colony, these were also classified as best. For quantitative analyses of switching, the visit rate was calculated for every run. Under our simulation conditions, the most profitable feeder always changed after 4 experimental hours. Thus, we calculated the visit rate at 4 and 8 hours separately. The visit rate was obtained by dividing the number of visits to each feeder during each phase by the total number of visits to all feeders during the corresponding phase, and then the obtained value was multiplied by 100 . Next, the switching index was used for examining colony responses to changes in the foraging environment. The switching index was calculated by subtracting the visit rate for the previously most profitable feeder, feeder 1 , from that for the currently most profitable feeder, feeder 2 , at the end of 
the simulation. Thus, the switching index ranged between -100 and 100 , and if the bees correctly switched feeders, the switching index was positive. The occurrence histograms for the switching index were created by counting the number of cases for every switching index of 10 (for variation) or 20 (for a combination of variation and distance). For the detailed analysis of switching indices, switching was separately analyzed for each combination of variations and distances. All switching indices from the 2-, 5-, 7-, and 10-feeder condition for a particular variation-distance combination were pooled. The degree of advantage conferred by the waggle dances for each combination was calculated by counting the number of sub-conditions (each combination consisted of 4 sub-conditions, because simulations were done for 4 different numbers of feeders) under which the waggle dance colony was the best. If the waggle dance colony was the best under all sub-conditions for a particular combination, the degree of advantage was 4. In contrast, a degree of 0 indicated that there were no sub-conditions under which the waggle dance colony was the best for that combination.

Simulations and analysis were carried out using all possible combinations of the 4 different numbers of feeders, 7 distances, and 6 variations. Full Methods and all associated references are provided in the Supplementary Methods.

Statistics. All statistical analyses of colony effects were performed using Analysis of Variance (ANOVA) and a post-hoc Tukey-Kramer test unless otherwise mentioned.

1. von Frisch, K. The Dance Language and Orientation of Bees. (Harvard University Press, Cambridge, 1993).

2. Gould, J. M. \& Gould, C. G. The Honey Bee. (Scientific American Library, New York, 1988).

3. Seeley, T. The Wisdom of the Hive. (Harvard University Press, Cambridge, 1995).

4. Beekman, M., Doyen, L. \& Oldroyd, B. P. Increase in dance imprecision with decreasing foraging distance in the honey bee Apis mellifera $L$. is partly explained by physical constraints. J. Comp. Physiol. [A] 191, 1107-1113 (2005).

5. Weidenmüller, A. \& Seeley, T. D. Imprecision in waggle dances of the honeybee (Apis mellifera) for nearby food sources: error or adaptation? Behav. Ecol. Sociobiol. 46, 190-199 (1999).

6. Tanner, D. A. \& Visscher, K. Do honey bees tune error in their dances in nectarforaging and house-hunting? Behav. Ecol. Sociobiol. 59, 571-576 (2006).

7. Tanner, D. A. \& Visscher, K. Do honey bees average directions in the waggle dance to determine a flight direction? Behav. Ecol. Sociobiol. 62, 1891-1898 (2008).

8. Meikle, W. G., Rector, B. G., Mercadier, G. \& Holst, N. Within-day variation in continuous hive weight data as a measure of honey bee colony activity. Apidologie 39, 694-707 (2008).

9. Okada, R., Ikeno, H., Aonuma, H. \& Ito, E. Biological insights into robotics: honeybee foraging behavior by waggle dance. Adv. Robotics 22, 1665-1681 (2008).

10. Okada, R. et al. The dance of the honeybee: how do they dance to transfer the food information effectively? Acta. Biol. Hung. 59 (suppl), 157-162 (2008).

11. De Marco, R., Gurevitz, J. M. \& Menzel, R. Variability in the encoding of spatial information by dancing bees. J. Exp. Biol. 211, 1635-1644 (2008).

12. Visscher, P. K. \& Seeley, T. D. Foraging strategy of honeybee colonies in a temperate deciduous forest. Ecology 63, 1790-1801 (1982).

13. Carrión-Tacuri, J. et al. Nectar production by invasive Lantana camara and Endemic L. peduncularis in the Galápagos islands. Pacific Science 66, 435-445 (2012).

14. Mačukanovič, M. \& Blaženčič, Ž. Diurnal and seasonal dynamics of nectar secretion of some species in the family Lamiaceae. Acta. Vet. 48, 173-183 (1998).

15. Farkas, Á. \& Orosz-Kovács, Z. s. Nectar secretion dynamics of Hungarian local pear cultivars. Plant. Syst. Evol. 238, 57-67 (2003).

16. Srinivasan, M. V. Honeybee communication: a signal for danger. Curr. Biol. 20, R366-R368 (2010).

17. Okada, R. et al. Waggle dance effect: dancing in autumn reduces the weight loss of a honeybee colony. J. Exp. Biol. 215, 1633-1641 (2012).

18. Dornhaus, A. \& Chittka, L. Why do honey bees dance? Behav. Ecol. Sociobiol. 55 395-401 (2004).

19. Sherman, G. \& Visscher, K. P. Honeybee colonies achieve fitness through dancing. Nature 419, 920-922 (2002).

20. Seeley, T. D., Camazine, S. \& Sneyd, J. Collective decision-making in honey bees: how colonies choose among nectar sources. Behav. Ecol. Sociobiol. 28, 277-290 (1991).

21. Couvillon, M. J., Philipps, H. L. F., Schürch, R. \& Ratnieks, F. L. W. Working against gravity: horizontal honeybee waggle runs have greater angular scatter than vertical waggle runs. Biol. Lett. 8, 540-543 (2012).

22. Tanner, D. A. \& Visscher, P. K. Adaptation or constraint? Reference-dependent scatter in honey bee dances. Behav. Ecol. Sociobiol. 64, 1081-1086 (2010).

23. Dyer, F. C. The biology of the dance language. Annu. Rev. Entomol. 47, 917-949 (2002).

24. Tanner, D. A. \& Visscher, P K. Does imprecision in the waggle dance fit patterns predicted by the tuned-error hypothesis? J. Insect Behav. 23, 180-188 (2010)

25. Ohtani, T. An Ethological Study of Adult Female Honeybees within the Hive. Sapporo, Japan: PhD Thesis, Hokkaido University (1986).

26. Seeley, T. D. Honey bee foragers as sensory units of their colonies. Behav. Ecol. Sociobiol. 34, 51-62 (1994).
27. De Marco, R. How bees tune their dancing according to their colony's nectar influx: re-examining the role of the food-receivers' 'eagerness'. J. Exp. Biol. 209, 421-432 (2006)

28. Cox, M. D. \& Myerscough, M. R. A flexible model of foraging by a honey bee colony: the effects of individual behaviour on foraging success. J. Theor. Biol. 223, 179-197 (2003)

29. Camazine, S. \& Sneyd, J. A model of collective nectar source selection by honeybee: Self-organization through simple rules. J. Theor. Biol. 149, 547-571 (1991).

30. de Vries, H. \& Biesmeijer, J. C. Modelling collective foraging by means of individual behaviour rules in honey-bees. Behav. Ecol. Sociobiol. 44, 109-124 (1998).

31. Johnson, B. R. \& Nieh, J. C. Modeling the adaptive role of negative signaling in honey bee intraspecific competition. J. Insect Behav. 23, 459-471 (2010).

32. Schmickl, T. \& Crailsheim, K. Costs of environmental fluctuations and benefits of dynamic decentralized foraging decisions in honey bees. Adapt. Behav. 12, 23-277 (2004).

33. Towne, W. F. \& Gould, J. L. The spatial precision of the honey bee's dance communication. J. Insect Behav. 1, 129-155 (1988).

34. Beekman, M. \& Lew, J. B. Foraging in honeybees-when does it pay to dance? Behav. Ecol. 19, 255-262 (2008).

35. Granovskiy, B., Latty, T., Duncan, M., Sumpter, D. J. T. \& Beekman, M. How dancing honey bees keep track of changes: the role of inspector of bees. Behav. Ecol. 23, 588-596 (2012).

36. Dornhaus, A., Klügl, F., Oechslein, C., Puppe, F. \& Chittka, L. Benefits of recruitment in honey bees: effects of ecology and colony size in an individualbased simulation model. Behav. Ecol. 17, 336-344 (2006).

37. Sumpter, D. J. T. \& Pratt, S. C. A modelling framework for understanding social insect foraging. Behav. Ecol. Sociobiol. 53, 131-144 (2003).

38. Okada, R. et al. Mathematical analysis of the honeybee waggle dance. Acta. Biol. Hung. 63 (suppl. 2), 201-205 (2012).

39. Okada, R. et al. Markov model of the honeybee social behavior. Information 13, 1115-1130 (2010).

40. Seeley, T. D. et al. Stop signals provide cross inhibition in collective decisionmaking by honeybee swarms. Science 335, 108-111 (2012).

41. Martin, H. \& Lindauer, M. Der Einflu $\beta$, des Erdmagnetfeldes auf die Schwereorientierung der Honigbiene (Apis mellifica). J. Comp. Physiol. 122, 145-187 (1977)

42. Menzel, R. et al. A common frame of reference for learning and communicated vectors in honeybee navigation. Curr. Biol. 21, 645-650 (2011).

43. Grüter, C., Moore, H., Firmin, N., Helanterä, H. \& Ratnieks, F. L. W. Flower constancy in honey bee workers (Apis mellifera) depends on ecologically realistic rewards. J. Exp. Biol. 214, 1397-1402 (2011).

44. Michelsen, A., Andersen, B. B., Kirchner, W. H. \& Lindauer, M. Honeybees can recruited by a mechanical model of a dancing bee. Naturwissenchaft. 76, 277-280 (1989).

45. Moss, F. \& Wiesenfeld, N. The benefits of background noise. Sci. Am. 273, 50-53 (1995).

46. Russell, D. F., Wilkens, L. A. \& Moss, F. Use of behavioural stochastic resonance by paddle fish for feeding. Nature 402, 291-294 (1999).

47. Shimozawa, T., Murakami, J. \& Kumagai, T. Cricket wind receptors: thermal noise for the highest sensitivity known. Sensors and Sensing in Biology and Engineering Barth, F. G., Humphrey, J. A. C. \& Secomb, T. (eds.), 145-157 (Springer-Verlag, Berlin, 2003).

48. Levin, J. E. \& Miller, J. P. Broadband neural encoding in the cricket cercal sensory system enhanced by stochastic resonance. Nature 380, 165-168 (1996)

49. Douglass, J. K., Welkens, L., Pantazelou, E. \& Moss, F. Noise enhancement of information transfer in crayfish mechanoreceptors by stochastic resonance. Nature 365, 337-340 (1993).

50. Dussutour, A., Beekman, M., Nicolis, S. C. \& Meyer, B. Noise improves collective decision-making by ants in dynamic environments. Proc. R. Soc. B 276, 4353-4361 (2009)

51. De Marco, R. J. \& Farina, W. M. Change in food source profitability affect the trophallactic and dance behavior of forager honeybees (Apis mellifera L.). Behav. Ecol. Sociobiol. 50, 441-449 (2001).

\section{Acknowledgments}

This research was supported by Grants-in-Aid for Scientific Research on Priority Areas "Emergence of Adaptive Motor Function through Interaction between Body, Brain and Environment" from the Japanese Ministry of Education, Culture, Sports, Science and Technology (area No. 454 to H.A., and grant nos. 18047003 and 20033023 to E.I.)

\section{Author contributions}

R.O. planned and carried out all the biological and simulation experiments, analyzed the data, and prepared the figures. R.O., H.I. and E.I. created the model. R.O., H.I., T.K., M.O., H.A. and E.I. interpreted the results and wrote the paper. 


\section{Additional information}

Supplementary information accompanies this paper at http://www.nature.com/ scientificreports

cc)(1) This work is licensed under a Creative Commons Attribution-

Competing financial interests: The authors declare no competing financial interests. visit http://creativecommons.org/licenses/by-nc-sa/3.0

How to cite this article: Okada, R. et al. Error in the Honeybee Waggle Dance Improves Foraging Flexibility. Sci. Rep. 4, 4175; DOI:10.1038/srep04175 (2014). 